\title{
ERRATUM
}

Parvaneh Keshavarz $\cdot$ Hiroshi Inoue $\cdot$ Yukiko Sakamoto

Kiyoshi Kunika · Toshihito Tanahashi

Naoto Nakamura · Toshikazu Yoshikawa

Natsuo Yasui $\cdot$ Hiroshi Shiota $\cdot$ Mitsuo Itakura

\section{No evidence for association of the ENPP1 (PC-1) K121Q variant with risk of type 2 diabetes in a Japanese population}

Published online: 22 June 2006

(C) The Japan Society of Human Genetics and Springer-Verlag 2006

\section{J Hum Genet (2006) 51:559-566}

The received date that was shown for this article was incorrect. It should read 31 January, 2006.

The online version of the original article can be found at http:// dx.doi.org/10.1007/s10038-006-0399-0

P. Keshavarz $\cdot$ H. Inoue $(\bowtie) \cdot$ Y. Sakamoto

K. Kunika $\cdot$ T. Tanahashi $\cdot$ M. Itakura

Division of Genetic Information,

Institute for Genome Research,

The University of Tokushima,

3-18-15 Kuramoto-cho, Tokushima 770-8503, Japan

E-mail: hinoue@genome.tokushima-u.ac.jp

Tel.: + 81-88-6339483

Fax: + 81-88-6339484

N. Nakamura $\cdot$ T. Yoshikawa

Department of Endocrinology and Metabolism,

Kyoto Prefectural University of Medicine,

Graduate School of Medical Science, Kyoto, Japan

N. Yasui

Department of Orthopedics, Institute of Health Biosciences,

The University of Tokushima, Tokushima, Japan

H. Shiota

Department of Ophthalmology and Visual Neuroscience,

Institute for Health Biosciences, The University of Tokushima,

Tokushima, Japan 